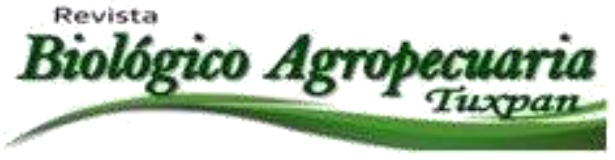

\title{
Fluctuación poblacional de la escama blanca del mango (Aulacaspis tubercularis Newstead) en Veracruz, México
}

Population fluctuations of the handle white flake (Aulacaspis tubercularis Newstead) in Veracruz, Mexico

Montiel-Vicencio Gerardo ${ }^{1 凶}$, Mario Alfonso Urías-López ${ }^{2}$ y Nain Peralta-Antonio ${ }^{1}$

${ }^{1}$ INIFAP. Campo Experimental Cotaxtla. Km. 34.5 Carretera Federal. Veracruz-Córdoba, Mpio. Medellín de Bravo, Veracruz. C.P. 94270 México. ${ }^{2}$ INIFAP. Campo Experimental Santiago Ixcuintla. Carretera. Internacional México-Nogales, km. 6 entronque a Santiago Ixcuintla. C.P. 63300. Santiago Ixcuintla, Nayarit; México.

${ }^{\square}$ Autor para correspondencia: montiel.gerardo@inifap.gob.mx

Recibido: 07/01/2014

Aceptado: 14/07/2014

\section{RESUMEN}

Los estudios se realizaron, de 2012 a 2013 en huertos comerciales de mango manila en tres localidades de Veracruz, México; (Actopan, Cotaxtla y Tierra Blanca); con el objetivo de obtener información sobre la fluctuación de población anual de la escama blanca del mango. El monitoreo de las poblaciones se realizó cada quince días, en cada huerto se muestrearon cinco árboles a los cuales se marcaron cuatro ramas con orientación a cada punto cardinal. Se encontró que la especie que ataca al mango en Veracruz es Aulacaspis tubercularis Newstead (Hemíptera: Diaspididae), misma que se presenta en Nayarit, México. Respecto a la fluctuación poblacional se observaron dos eventos de crecimiento poblacional una de baja densidad desde el mes de julio periodo de lluvias, hasta el mes de enero y la segunda de incremento poblacional floreció de febrero a mayo, sin embargo en marzo se detectaron diferencias significativas en las tres localidades en el número de escamas (hembras), el promedio significativamente más alto por hoja (11.32) se registró en Actopan, seguido por Tierra Blanca (4.27) y finalmente en Cotaxtla (0.89). En cuanto a las colonias de escama (machos) se detectaron diferencias significativas en marzo; Actopan registró la infestación más alta de colonias por hoja (4.12), y la más baja Cotaxtla (0.89) superado por Tierra Blanca (2.92); los resultados muestran que las tres localidades fueron susceptibles a la escama blanca.

Palabras claves: escama blanca, Aulacaspis tubercularis, mango. 


\section{ABSTRACT}

The studies were conducted from 2012-2013 in manila mango commercial orchards in three locations in Veracruz, Mexico; (Actopan, Cotaxtla and Tierra Blanca), with the aim of obtaining information on the annual population fluctuation white flake handle. The population monitoring was performed every fifteen days; in each orchard were sampled five trees which marked the four branches facing each cardinal point. It was found that the species that attacks the handle is Aulacaspis tubercularis Veracruz Newstead (Hemiptera: Diaspididae), same as presented in Nayarit, Mexico. Regarding population fluctuation two events were observed population growth of low density from July rainy season until January and the second in population growth flourished from February to May, however in March were no significant differences at the three localities in the number of scales (females), significantly higher average per sheet (11.32) was recorded in Actopan, followed by Tierra Blanca (4.27) and finally in Cotaxtla (.89). Regarding flake colonies (males) significant differences were detected in March in Actopan infestation recorded highest colonies per sheet (4.12), and the lowest in Cotaxtla (0.89), Tierra Blanca overcome by (2.92), the results show that the three sites were susceptible to white scale.

Keywords: white flake, Aulacaspis tubercularis, mango.

\section{INTRODUCCIÓN}

El mango es uno de los cultivos tropicales más importantes a nivel mundial. México ocupa el cuarto lugar con el $6 \%$ en producción después de Tailandia (McCoy, 2007). Veracruz es el principal productor de mango manila con 21,154.21 ha, sin embargo ocupa el sexto lugar por superficie sembrada (SIAP, 2013). El mango es atacado por numerosas plagas, entre las que destacan, las moscas de la fruta, considerada como plaga de mayor importancia comercial (Aluja, 1993); sin embargo la escama blanca es otra de las principales plagas que atacan el cultivo del mango; en México fue encontrada por primera vez en 2003; en Compostela, Nayarit. (Urías et al. 2010); los daños que provoca esta plaga son directos en la calidad del fruto ya que provoca manchas cloróticas, además las ninfas se alimentan de la savia de las hojas, causando caída prematura de éstas; en Veracruz la escama blanca genera un rechazo de los frutos por parte de los consumidores del 30\%; en comparación con Nayarit, en donde el rechazo del fruto es superior al $50 \%$ en las empacadoras (Urías, 2006). Las hembras A. tubercularis son circulares, delgadas y planas cubierta por una membrana blanca; los machos son mas pequeños de color blanco regularmente se encuentran en colonias (Hodges y Hamon, 2006), citado por Urías et al. (2010). Por lo importante y representativo que es el cultivo del mango en Veracruz, el objetivo del presente trabajo de investigación fue generar información sobre la fluctuación poblacional anual de la escama blanca en las localidades (Actopan, Tierra Blanca y Cotaxtla).

\section{MATERIALES Y MÉTODOS}

Los estudios se realizaron, de junio 2012 a mayo 2013, en huertos comerciales de mango manila de tres localidades del estado de Veracruz, México, (Actopan, Cotaxtla y Tierra Blanca); la técnica de muestreo que se ocupó 
durante los monitoreos fue la utilizada por Urías et al. (2010), se realizaron principalmente muestreos cada quince días en cada huerto, de cada uno se seleccionaron cinco arboles de tamaño similares, a los cuales se les marcaron cuatro ramas con orientación a cada punto cardinal; dentro de cada rama se marcaron los penúltimos flujos vegetativos y se tomaron en cuenta dos hojas izquierda y derecha (1 y 2) respectivamente, para obtener un total de 8 hojas/árbol por muestreo. En cada muestreo se registró como variables de estudio el número de colonias (grupo de machos) y hembras presentes en haz y envés de las hojas. El diseño experimental utilizado para este trabajo fue bloques completamente al azar con cinco repeticiones (árboles), cada árbol se utilizó como unidad experimental, los datos de analizaron con el programa estadístico de SAS 9.2 y la comparación de medias mediante la prueba de Tukey $(\mathrm{P} \leq 0.05)$.

\section{RESULTADOS Y DISCUSIÓN}

En las tres localidades del cultivar manila, los análisis estadísticos mostraron diferencias significativas de escama blanca hembras $(\mathrm{H})$ y colonias de machos (C.M) registradas durante junio 2012 a mayo 2013; (Fig. 1y 2). En Actopan se registraron bajas poblaciones de escama blanca en agosto y septiembre $2012 \quad(0.97 \quad$ y $1.05 \quad$ H/hoja $)$ respectivamente; pero en promedio se observó un incremento en febrero 2013 (3.12 H/hoja), hasta alcanzar el pico mas alto en marzo (11.32 $\mathrm{H} /$ hoja). En Cotaxtla los valores registraron una población baja en todo el año (0.82 y 0.87

H/hojas), en septiembre y enero respectivamente. Respecto a Tierra Blanca la menor cantidad se registró en enero (0.5 H/hojas) y el mas alto (4.27 H/hojas) se registraron en marzo 2013 (Fig. 1).

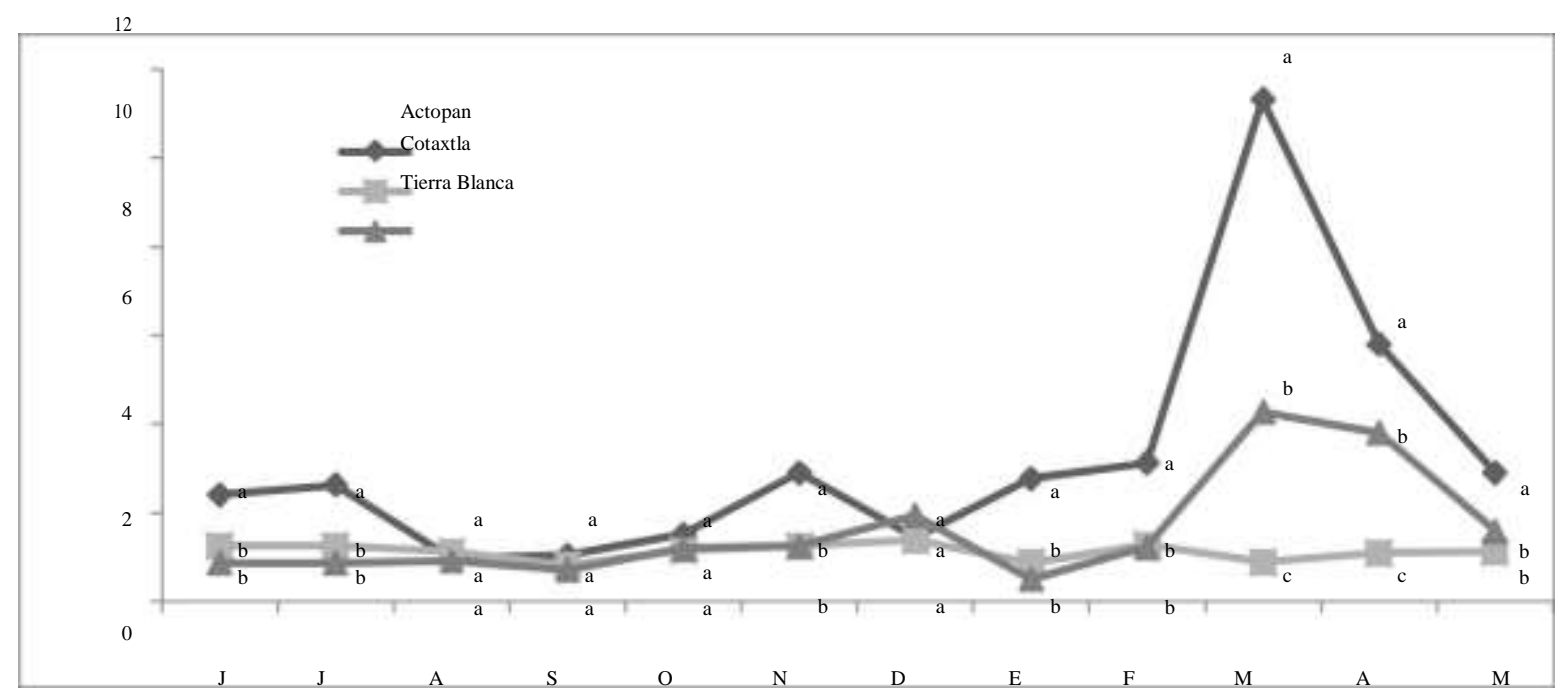

Figura 1. Fluctuación poblacional de la escama blanca (hembras) Aulacaspis tubercularis en mango manila, 2012-2013. Veracruz.

Respecto a la fluctuación de escama de colonias de machos (C.M); en Actopan el punto más bajo se observó en junio (0.60 C.M/hoja) y el mas alto en marzo (4.12 C.M/hoja). En 
Cotaxtla el promedio en colonias mas bajo fue en agosto (0.55 C.M/hoja), sin embargo el máximo fue en febrero con (1.82 C.M/hoja).

Para Tierra Blanca el punto mínimo (0.37 C.M/hoja) y máximo (2.92 C.M/hoja) se observó en agosto y marzo respectivamente (Fig. 2).

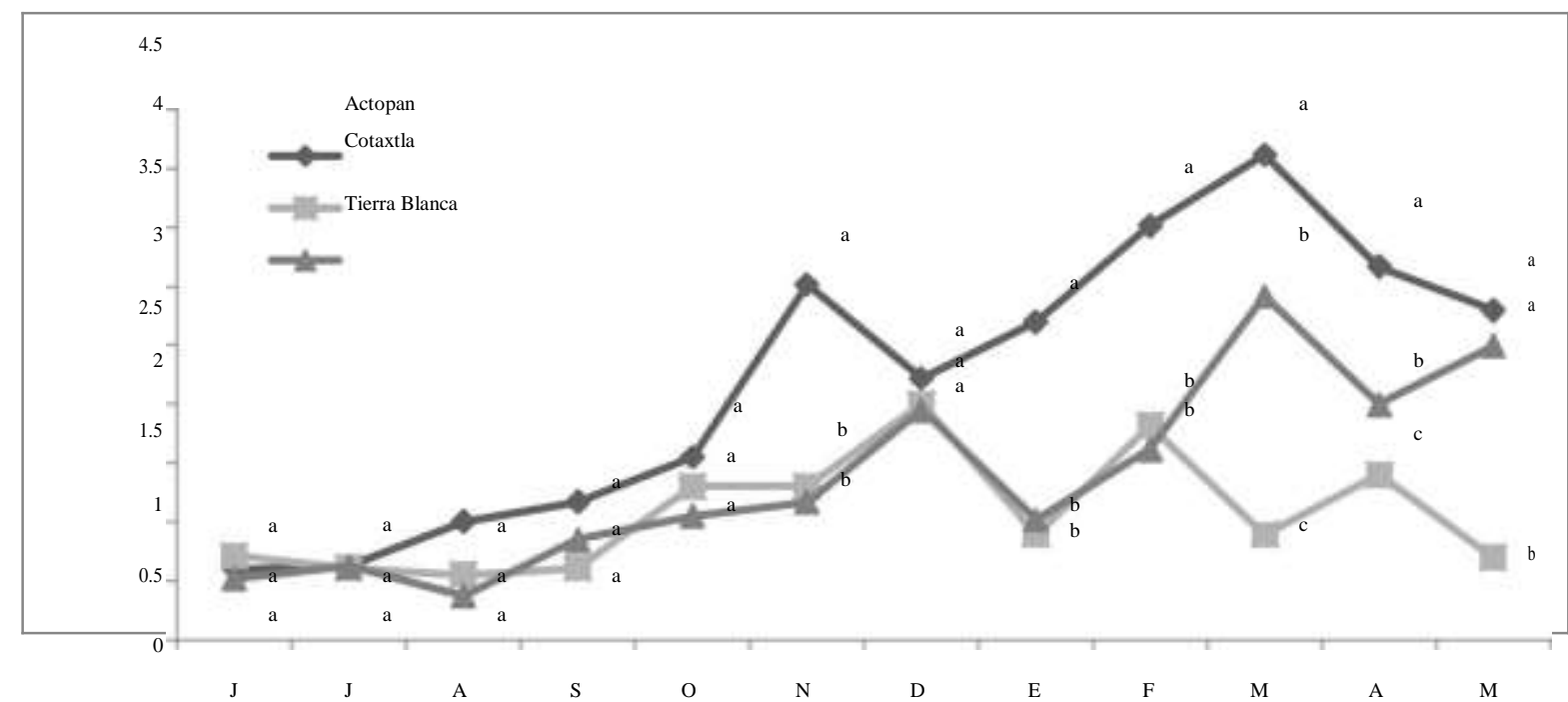

Figura 2. Fluctuación poblacional de la escama blanca (machos) Aulacaspis tubercularis en mango manila, 2012-2013. Veracruz.

\section{CONCLUSIONES}

El flujo de crecimiento de Aulacaspis tubercularis se comportó de diferente manera en las tres localidades; en general Cotaxtla fue el que menos infestación de escama blanca tuvo, los valores mas altos que se observaron fueron en diciembre (1.38 H/hoja) y (1.95 C.M/hoja). La segunda se observa en Tierra Blanca que tuvo una infestación media respecto a los demás. El pico mas alto fue en marzo (11.32 H/hoja) y (4.12 C.M/hoja), siendo Actopan el que mostró mayor población. Finalmente los resultados indican que las tres localidades fueron susceptibles a la escama blanca.
Esta investigación es financiada por CONACYT.

\section{LITERATURA CITADA}

Aluja, M. 1993. Manejo integrado de las moscas de la fruta. Editorial trillas, México DF.

McCoy, S. 2007. Organic mangoes: a production guide.Bulletin 4712.Department of Agriculture and Food, Western Australia.42 pp.

SIAP-SAGARPA. 2013. Anuario estadístico de la producción agrícola. SIAP. México.

En línea. http://www.mango.gob.mx/index.php? AGRADECIMIENTOS portal=mango . Consultado el 29 de julio de 2013.

\section{Revista Científica Biológico Agropecuaria Tuxpan 2 (1)}


Urías, L. M. A. 2006. Principales plagas del mango en Nayarit. In: El cultivo del mango: Principios y tecnología de producción. V. Vázquez V. y M. H. Pérez B. (Eds.). Instituto Nacional de Investigaciones Forestales, Agrícolas y Pecuarias. Santiago Ixcuintla, Nayarit. pp. 211-234. https://doi.org/10.5154/r.rchsh.2009.15.017
Montiel et al., 2014

Urías-López, M. A., Osuna-García, J. A., Vázquez-Valdivia, V. y Pérez-Barraza, M. H. 2010. Fluctuación poblacional y distribución de la escama blanca del mango (Aulacaspis tubercularis Newstead) en Nayarit, México. Revista Chapingo serie Horticultura.16 (2): 77-82. https://doi.org/10.5154/r.rchsh.2010.16.009

Copyright (c) 2014 Gerardo Montiel Vicencio, Mario Alfonso Urias López yNain Peralta Antonio

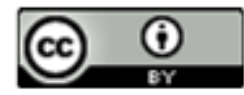

Este texto está protegido por una licencia Creative Commorr 4.0

Usted es libre para Compartir —copiar y redistribuir el material en cualquier medio o formato- y Ad aptar el documento —remezclar, transformar y crear a partir del material- para cualquier propósito, inchso para fines comerciales, siempre que cumpla la condición de:

Atribución: Usted debe dar crédito a la obra original de manera adecuada, proporcionar un enlace a la licencia, e indicar si se han realizado cambios. Puede hacerlo en cualquier forma razonable, pero no de forma tal que sugiera que tiene el apoyo del licenciante olo recibe por el usoque hace de la obra.

Resumendelicencia - Textocompletodelalicencia 